\title{
A THERMODYNAMIC THEORY FOR A RESTRICTED CLASS OF CONTACT BEHAVIOUR
}

\author{
F. J. M. Starmans, $\dagger$ W. A. M. Brekelmans and J. D. Janssen \\ Department of Mechanical Engineering, Eindhoven University of Technology, The Netherlands
}

(Received 17 January 1989 ; in revised form 2 November 1989)

\begin{abstract}
A thermodynamic continuum theory for contact behaviour under mild forming conditions is presented. The modelling of the contacts, balance laws, dependent and independent variables as well as entropy inequality are considered. It is shown that Maxwell friction models can be derived from the thermodynamic framework.
\end{abstract}

\section{INTRODUCTION}

The Coulomb and Von Mises friction models have for many decades been applied to describe the mechanical interaction between die and workpiece in forming processes. As these models only cover experimental results to a certain degree, extended friction models analogous to material models of the Maxwell type have been proposed for instance by Fredriksson (1976), Petersson (1977), Michalowski and Mróz (1978), Cheng and Kikuchi (1985) and Baaijens et al. (1986). In the present paper a thermodynamic continuum theory for contact behaviour is set up and Maxwell friction models, as well as thermodynamic restrictions for their validity, are derived from this framework. The first impulses to thermodynamic theories for contact behaviour were given by Bikerman (1970) and Klamecki (1980). A very comprehensive theory has been formulated by Zmitrowicz (1987). In the present instance we restrict ourselves to a theory for contact processes under mild forming conditions, defined by the following set of restrictions :

-quasi-static processes. Negligible inertial effects, absence of big temperature changes due to external sources;

-no wear or fretting. Wear particles or conditions of grave material transference between the contact partners are avoided, resulting in only two body-contact situations;

-moderate forming contact pressures. Only one of the contact partners shows large, irreversible deformations;

-non-aggressive physical and chemical conditions in immediate environment ;

-initial homogeneity at the contact surface. The conditions of lubrication and roughness are the same at all contact points before loading is applied. Hydrodynamic lubrication is not considered.

In the following sections the modelling of contact regions within continuum theory is first discussed and balance laws are formulated. The complexity of these laws is reduced by the introduction of some simplifying assumptions and specific application to the class of contact processes under mild forming conditions. Distinction between independent and dependent variables and application of some constitutive principles yield a set of constitutive contact equations. In order to derive the Maxwell friction laws, internal variables and evolution laws for these variables are introduced so that thermodynamic requirements are met. Experiments are needed for investigation of their applicability in engineering practice, an aspect not further considered here. 


\section{DESCRIPTION OF CONTACT REGIONS IN CONTINUUM THEORY}

In literature, the description of real contact phenomena by means of two smooth body surfaces, possibly via an intermediate surface or layer, is generally introduced more or less intuitively (Oden and Martins, 1985: Ruina, 1985). In this section, the concept of averaging volumes is used to support this intuitive process mathematically. Better understanding is thus obtained as to the validity of the particular modelling of contact regions.

Two contacting bodies are considered, mechanically and thermally loaded. see Fig. 1. Phenomena occur within and between the bodies. It is assumed that the insides of the bodies may be modelled as continua. In the transition region between the bodies. capricious contacts result from the roughness of both. In this region, chemically and physically different components are present, such as oxide layers, lubricant and wear particles (Rabinowicz, 1965). The resulting macroscopic behaviour caused by the interaction on a microscopic scale, can be ascribed to a fictitious smooth contact layer between the two bodies. Phenomena such as slip, contact stiffness and frictional heat can be described by such a layer (Oden and Martins, 1985; Zmitrowicz, 1987). It is noted that the roughness of the bodies is smoothed, and the influence of this is represented in the layer behaviour. A similar statement can be made as to the lubrication effects.

Although the microscopic behaviour need not be studied, definition of the contact layer and the ascription of continuum quantities to it starts at this point. A smooth nonunique midplane $\mathrm{S}$, situated in the transition region between the bodies, is momentarily assumed and elementary averaging volumes $V_{\mathrm{e}}$ introduced (Whitaker, 1969 ; Huyghe, 1986). The smoothness is defined with respect to local, i.e. microscopic, fluctuations. This will be quantified below. The points on the midplane $S$ are uniquely identified by two locational parameters $\eta_{1}$ and $\eta_{2}$ which compound a column $\eta$. For every point of S, denoted by $\eta$, a small cylindrical averaging volume $V_{\mathrm{e}}$ with diameter $D_{\mathrm{e}}$ and thickness $t_{\mathrm{c}}$ is defined, see Fig. 2. This volume is oriented so that the centre of $V_{\mathrm{e}}$ coincides with point $\eta$ of $\mathrm{S}$. and the axis of $V_{\mathrm{e}}$ is perpendicular to $\mathrm{S}$ at that point. The contact layer is defined as the region between the envelopes $S^{+}$and $S^{-}$of the averaging volumes for all points $\eta$. The curvature of $S$ inside $V_{\mathrm{e}}$ is neglected, which requires $D_{\mathrm{c}}$ to be much shorter than a characteristic radius of curvature of $\mathrm{S}$. The thickness of the contact layer thus equals $t_{\mathrm{e}}$ by approximation.

A quantity $\varphi$ which is defined for each material component in the transition region as a function of the position (and time) is considered. The surface average $\langle\varphi\rangle$ is introduced by

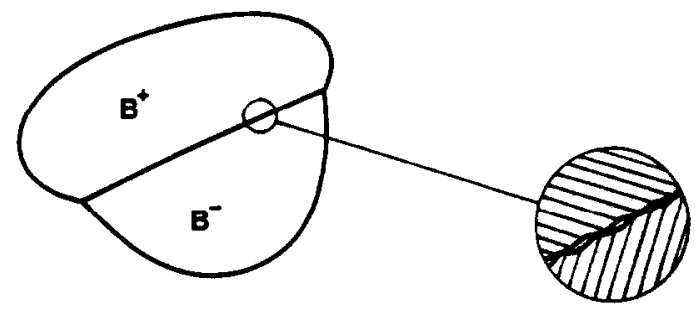

Fig. 1. Two contacting bodies $\mathrm{B}^{+}$and $\mathrm{B}^{-}$

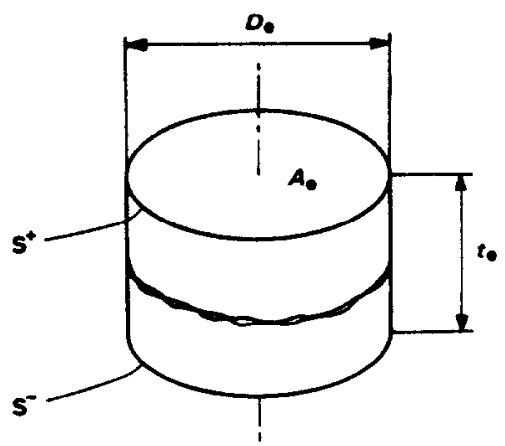

Fig. 2. Elementary averaging volume with a part of the contact region. 

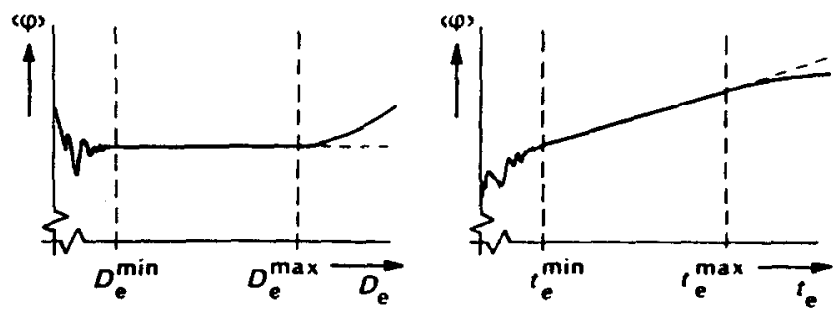

Fig. 3. The averages as functions of the diameter and thickness of the averaging volume.

$$
\langle\varphi\rangle=\frac{1}{A_{\mathrm{e}}} \int_{V_{\mathrm{c}}} \varphi \mathrm{d} V, \quad A_{\mathrm{e}}=\frac{\pi}{4} D_{\mathrm{e}}^{2}
$$

and is defined at each point $\eta$. At some time $t,\langle\varphi\rangle$ can be considered as a function of $\eta$, $D_{\mathrm{e}}$ and $t_{\mathrm{e}}$. To quantify the requirements for $D_{\mathrm{e}}$ and $t_{\mathrm{e}}$, the characteristic behaviour of $\langle\varphi\rangle$ for some $\eta$ and $t$, as a function of $D_{\mathrm{e}}$ and $t_{\mathrm{e}}$, is visualized in Fig. 3. Strong fluctuations occur below some $D_{\mathrm{e}}^{\min }$ and $t_{\mathrm{e}}^{\min }$. These fluctuations result from the roughness of both bodies and the distribution of components with strongly differing values of $\varphi$ through the transition region. In that lower range, the averages are useless as continuum quantities. Above some $D_{e}^{\max }$ and $t_{\mathrm{e}}^{\max }$, the averages are smooth, but diverge from the values which would occur under homogeneous thermomechanical loading conditions. This results from variation of $\varphi$ of one or more components influenced by global conditions. To ensure that this global behaviour is not filtered out, the upper range for the averaging volume dimensions should also be avoided. The averages based on volumes $V_{\mathrm{c}}$ with dimensions in the range between $D_{e}^{\min }, t_{\mathrm{e}}^{\min }$ and $D_{\mathrm{e}}^{\max }, t_{\mathrm{e}}^{\max }$ can be used as local continuum quantities. A continuum approach is not possible if such a range cannot be indicated. However, for contact problems in mechanical engineering such a range can generally be given. Suitable choices follow from

$$
d \ll D_{\mathrm{e}}, t_{\mathrm{e}} \ll L
$$

where $d$ is a characteristic distance for fluctuations in the local contact areas and $L$ a characteristic distance for global variations.

A number of kinematic relations is treated before balance laws are derived for the contact layer in the next section. In Fig. 4 a cross-section of a part of the contact layer is sketched. The points momentarily coinciding with the boundary surfaces $\mathrm{S}^{+}$and $\mathrm{S}^{-}$are identified uniquely by surface coordinates $\zeta^{+}$and $\zeta^{-}$. A point $\mathrm{P}$ of $\mathrm{S}$, with coordinates $\eta$, is associated with the points $\mathrm{P}^{+}$and $\mathrm{P}^{-}$of $\mathrm{S}^{+}$and $\mathrm{S}^{-}$, respectively. These points are located on a line perpendicular to $S$ at $P$. The coordinates $\zeta^{+}$and $\zeta^{-}$are thus immediately related to $\eta$. The position in space of point $\eta$ of $S$ at time $t$ is indicated by the vector $x_{s}=x_{s}(\eta, t)$

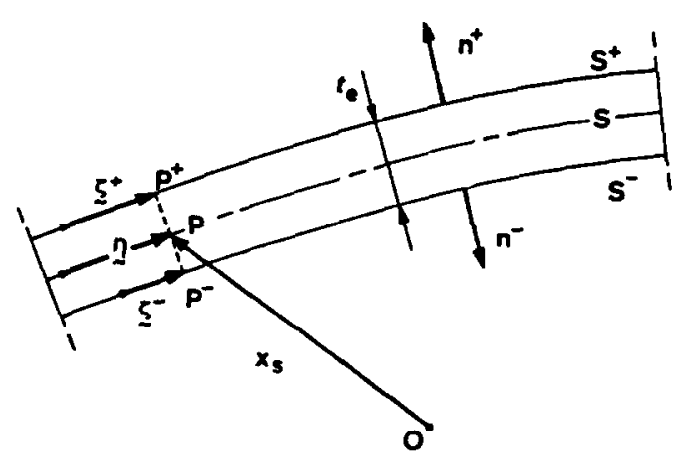

Fig. 4. The contact layer with definitions of quantities. 
or equivalently by $\mathbf{x}_{\mathrm{s}}=\mathbf{x}_{\mathrm{s}}\left(\xi^{+}, t\right)$ or $\mathbf{x}_{\mathrm{s}}=\mathbf{x}_{\mathrm{s}}\left(\zeta^{-}, t\right)$. Subsequently, only choices for the coordinates $\eta \cdot \xi^{+}$and $\xi^{-}$are considered, so that $\mathbf{x}_{\mathrm{s}}$ is continuous in $\eta$ and $t$, as well as in $\xi^{+}$ and $t$ or in $\xi$ and $t$. The natural and reciprocal surface base vectors $\mathfrak{c}_{s}$ and $\chi_{s}$ follow from

$$
\mathfrak{c}_{s}=\frac{\partial \mathbf{x}_{\mathrm{s}}}{\partial \eta}, \quad \mathbf{n}_{s}=\frac{\left(\mathbf{c}_{\mathrm{s}}\right)_{1} \times\left(\mathbf{c}_{\mathrm{s}}\right)_{2}}{\left\|\left(\mathbf{c}_{\mathrm{s}}\right)_{1} \times\left(\mathbf{c}_{\mathrm{s}}\right)_{2}\right\|}, \quad \mathbf{c}_{\mathrm{s}} \cdot \eta^{\mathrm{T}}=\underline{I}, \quad \chi_{s} \cdot \mathbf{n}_{\mathrm{s}}=\underline{0}
$$

where $\times$ denotes the outer (or cross) product, the dot $\cdot$ the inner (or dot) product, the superscript $T$ the transpose and $\|\mathbf{a}\|$ the magnitude of $\mathbf{a}$. $I$ is the unit matrix of rank 2 and $\underline{0}$ the zero column of length 2 . Since $S$ is a smooth surface, the unit normal vector $\mathbf{n}_{s}$ to $S$ is a continuous and differentiable function of $\eta$. The surface gradient operator $\nabla_{s}$ is defined by

$$
\nabla_{\mathrm{s}}=\chi^{\mathrm{T}} \frac{\hat{C}}{\hat{\partial} \underline{\underline{\eta}}}
$$

The surface velocity $\mathbf{u}_{\mathrm{s}}$ is defined as the velocity of point $\eta$ through space

$$
\mathbf{u}_{\mathrm{s}}=\mathbf{x}_{\mathrm{s}}^{\bullet}
$$

where the dot • denotes the time-derivative operator $\left.(\hat{c} / \partial t)\right|_{\eta}$.

\section{GENERAL BALANCE LAW AND SPECIFIC FORMULATIONS}

In this section the balance laws for mass, momentum, moment of momentum, energy and entropy are considered for the class of contacts defined in Section 1. These laws are special cases of the general balance law for some (mass-associated) quantity $\varphi$ which, for notational convenience, is assumed to be a scalar quantity. In order to derive this general formulation for $\varphi$, the quantity $Q$, defined for the averaging volume $V_{\mathrm{e}}$ pertaining to some point $\underline{\eta}$, is considered and is given by

$$
Q=\int_{V_{\mathrm{c}}} \rho \varphi \mathrm{d} V=A_{\mathrm{e}}\langle\rho \varphi\rangle
$$

where $\rho$ is the mass density. The quantity $Q$ may be identified physically, for instance the total mass of the material within $V_{\mathrm{e}}$, based on the choice $\varphi=1$. In that case the resulting balance law will express conservation of mass. Simplifications, valid for the restricted class of contacts, are indicated below. Essential assumptions, made to further simplify the balance laws, are discussed in the next section.

The time derivative $Q^{\bullet}$ of $Q$ is composed of contributions, brought about by material flow (convection), immaterial flow (flux) and sources, which will be reviewed in due course.

(1) Material flow through the border $\partial V_{\mathrm{e}}$ of $V_{\mathrm{e}}$

The local material velocity is indicated by $v$. The decrease in $Q$ per unit of time, due to convection, is thus

$$
\int_{\hat{c} v_{s}} \rho \varphi\left(\mathbf{v}-\mathbf{u}_{\mathrm{s}}\right) \cdot \mathbf{n} \mathrm{d} A
$$

where $\mathbf{n}$ denotes the unit outward normal vector. A uniform velocity $\mathbf{u}_{\mathrm{s}}$ is assumed for the whole boundary $\partial V_{\mathrm{e}}$. Formally this is incorrect if the orientation of $V_{\mathrm{e}}$ in space changes in time. These rotational effects may be neglected in view of the conditions in (2). 
(2) Flux through the boundary $\partial V_{\mathrm{e}}$

The local flux is indicated by $\psi$. The decrease in $Q$ resulting from fluxes is

$$
\int_{a v_{\mathrm{c}}} \mathrm{n} \cdot \boldsymbol{\psi} \mathrm{d} A
$$

(3) Sources within $V_{\text {. }}$

Here only the case of a distributed mass-associated $\varphi$-production $\Phi$ is considered. The increase in $Q$ is

$$
\int_{V_{\mathrm{c}}} \rho \Phi \mathrm{d} V
$$

Combination and elaboration of these three contributions, as given in Appendix A, result in the following general balance law for contact surfaces

$$
\begin{aligned}
\langle\rho \varphi\rangle^{\bullet}+\left(\nabla_{\mathrm{s}} \cdot \mathbf{u}_{\mathrm{s}}\right)\langle\rho \varphi\rangle & +\nabla_{\mathrm{s}} \cdot\left[\left(I-\mathbf{n}_{\mathrm{s}} \mathbf{n}_{\mathrm{s}}\right) \cdot\left(\left(\langle v \rho \varphi\rangle-\mathbf{u}_{\mathrm{s}}\langle\rho \varphi\rangle\right)+\langle\psi\rangle\right)\right]-\langle\rho \Phi\rangle \\
& =-\mathbf{n}^{+} \cdot\left(\psi^{+}+\left(\mathbf{v}^{+}-\mathbf{u}_{\mathrm{s}}\right) \rho^{+} \varphi^{+}\right)-\mathbf{n}^{-} \cdot\left(\psi^{-}+\left(\mathbf{v}^{-}-\mathbf{u}_{\mathrm{s}}\right) \rho^{-} \varphi^{-}\right) .
\end{aligned}
$$

The combination $\mathbf{n}_{\mathbf{s}} \mathbf{n}_{\mathbf{s}}$ is the dyadic product of $\mathbf{n}_{\mathbf{s}}$ with itself and $\boldsymbol{I}$ is the second-order unit tensor. The superscripts ${ }^{+}$and - indicate bulk continuum quantities at the + and - sides of the contact layer. Equation (10) is only valid if conditions (2) for the dimensions of the averaging volume are satisfied.

Some of the terms in (10) may be neglected or reformulated for the restricted class of contacts. Firstly, all sources can be neglected. The mechanical sources are negligibly small and the thermal ones are not present. Secondly, the contribution of the divergence of the in-plane fluxes is omitted. This is legitimate when the in-plane fluxes are small. For heat fluxes this will be true, as the conductance of contact layers is low in general, due to the presence of poor conducting components, such as lubricant and oxide layers. The in-plane surface stresses are generally not low. It is however expected that, because of the moderate forming-contact pressures, their gradient and divergence are of minor importance. In Sections 6 and 7 it will be seen that neglecting the influence of the surface stresses leads to constitutive relations which are unable to describe a sometimes physically observed effect. A third and final restriction is the exclusion of severe wear. This induces only slight mass flow from the bulk surroundings into the contact layer. The corresponding convection terms vanish from (10) by the adoption of $S^{+}$and $S^{-}$as material bulk surfaces, with material coordinates $\zeta^{+}$and $\zeta^{-}$, and surface averages based on a changing thickness $t_{\mathrm{e}}$, locally defined as the distance between the two material bulk surfaces. It is easily shown that (10) then becomes

$$
\langle\rho \varphi\rangle^{\bullet}+\left(\nabla_{s} \cdot u_{s}\right)\langle\rho \varphi\rangle+\nabla_{s} \cdot\left[\left(I-n_{s} n_{s}\right) \cdot\left(\langle v \rho \varphi\rangle-u_{s}\langle\rho \varphi\rangle\right)\right]=-n^{+} \cdot \psi^{+}-n^{-} \cdot \psi^{-}
$$

while the surface averages should be interpreted as averaged quantities of variable thickness. The change of the unit normal vectors $\mathbf{n}^{+}$and $\mathbf{n}^{-}$is a second-order effect and therefore these vectors can be taken perpendicular to $S$.

Special choices for $\varphi$ (and associated fluxes $\psi^{+}$and $\psi^{-}$) in (11) lead to the specific balance laws for the mass, momentum, moment of momentum, energy and entropy of $V_{\mathrm{e}}$. Mass. On substitution of $\varphi=1$, the quantity $Q$ represents mass

$$
\langle\rho\rangle+\left(\nabla_{s} \cdot u_{s}\right)\langle\rho\rangle+\nabla_{s} \cdot\left[\left(I-n_{s} n_{s}\right) \cdot\left(\langle v \rho\rangle-u_{s}\langle\rho\rangle\right)\right]=0 .
$$

Momentum. $\varphi$ is replaced by the material velocity $\mathbf{v}$, and the flux by the opposite of the conjugate $\Sigma^{*}$ of the Cauchy stress tensor $\Sigma$. Only non-polar media are considered so $\Sigma^{c}=\Sigma$. 
Neglecting the momentum, there being interest only in quasi-static processes, the static equilibrium equation results in

$$
n^{+} \cdot \Sigma^{+}+n^{-} \cdot \Sigma^{-}=0 .
$$

This equation represents the law of action and reaction between the two bodies.

Moment of momentum. For non-polar media the static moment equilibrium equation yields

$$
\mathbf{x}^{+} \times\left(\Sigma^{+} \cdot \mathbf{n}^{+}\right)+\mathbf{x}^{-} \times\left(\Sigma^{-} \cdot \mathbf{n}^{-}\right)=\mathbf{0} .
$$

Use of (13) in (14) offers a non-trivial equation. Because $t_{\mathrm{c}} \ll L$ this remaining equation describes a second-order effect. As the first-order effect is automatically satisfied by the static equilibrium equation, the static moment equation is not considered further.

Energy. The relevant material coupled energy is the internal energy e, the kinetic energy being negligible for quasi-static processes. The flux consists of a mechanical part $-\mathbf{v} \cdot \Sigma$ and a thermal part $\mathbf{q}$, equal to the heat flux in the case considered. The balance equation for the internal energy obtained is

$$
\begin{aligned}
& \langle\rho e\rangle^{\bullet}+\left(\nabla_{s} \cdot u_{s}\right)\langle\rho e\rangle+\nabla_{s} \cdot\left[\left(I-n_{s} n_{s}\right) \cdot\left(\langle\rho v e\rangle-u_{s}\langle\rho e\rangle\right)\right]=-n^{-} \cdot\left(q^{-}-v^{+} \cdot \Sigma^{+}\right) \\
& -\mathbf{n}^{-} \cdot\left(\mathbf{q}^{-}-\mathbf{v}^{-} \cdot \mathbf{\Sigma}^{-}\right) \text {. }
\end{aligned}
$$

The bulk of the bodies exchanges heat with the contact layer, but also performs on it work which is used for deformation in the thickness and shear directions. The latter results in macroscopic slip between the bodies, for which the work needed will be dissipated almost completely as frictional heat in the contact layer.

Entropy. A Clausius-Duhem equation for the entropy production $s$ is derived with the general balance law formulation

$$
\langle\rho s\rangle=\langle\rho h\rangle+\left(\nabla_{\mathrm{s}} \cdot \mathbf{u}_{\mathrm{s}}\right)\langle\rho h\rangle+\nabla_{\mathrm{s}} \cdot\left[\left(I-\mathbf{n}_{\mathrm{s}} \mathbf{n}_{\mathrm{s}}\right) \cdot\left(\langle\mathbf{v} \rho h\rangle-\mathbf{u}_{\mathrm{s}}\langle\rho h\rangle\right)\right]+\mathbf{n}^{+} \cdot \mathbf{w}^{-}+\mathbf{n}^{-} \cdot \mathbf{w}^{-}
$$

where $h$ is the entropy and $w$ the entropy flux. The second law of thermodynamics postulates $\langle\rho s\rangle=0$ for all allowable reversible contact processes and $\langle\rho s\rangle>0$ for all thermodynamically allowable irreversible contact processes. The term thermodynamically allowable indicates that the processes do not violate other thermodynamic concepts, such as the balance of internal energy, for example.

\section{SIMPLIFYING ASSUMPTIONS}

Assumptions are introduced for some quantities in the equations, as formulated in the previous section, in order to simplify the balance laws further.

First the flow terms are considered. In contrast to the flux terms in the previous section, the flow terms in the contact plane cannot simply be neglected. Choosing the surface coordinates $\eta$ so that $\left(I-\mathbf{n}_{s} \mathbf{n}_{\mathrm{s}}\right) \cdot\left(\langle\mathbf{v} \rho\rangle-\mathbf{u}_{\mathrm{s}}\langle\rho\rangle\right)=\mathbf{0}$, the term $\nabla_{\mathrm{s}} \cdot\left[\left(I-\mathbf{n}_{s} \mathbf{n}_{\mathrm{s}}\right) \cdot\left(\langle\mathbf{v} \rho\rangle-\mathbf{u}_{\mathrm{s}}\langle\rho\rangle\right)\right]$ disappears from the balance of mass. The similar terms in the balance of energy and the balance of entropy vanish with the additional assumption

$$
\left(I-\mathbf{n}_{\mathrm{s}} \mathbf{n}_{\mathrm{s}}\right) \cdot\left(\langle v \rho e\rangle-\mathbf{u}_{\mathrm{s}}\langle\rho e\rangle\right) \approx\left(I-\mathbf{n}_{\mathrm{s}} \mathbf{n}_{\mathrm{s}}\right) \cdot\left(\langle\mathbf{v} \rho h\rangle-\mathbf{u}_{\mathrm{s}}\langle\rho h\rangle\right) \approx 0 .
$$

In order to choose the surface coordinates as indicated, the physical quantities $\left(I-n_{s} n_{s}\right) \cdot\langle v \rho\rangle$ and $\langle\rho\rangle$ have to be known. In the case of mild forming conditions with only one contact partner undergoing considerable deformations, an approximate choice for $\eta$ can be made. If the averaging volume mainly contains part of the deforming contact 
partner, arbitrarily chosen to be body $B^{+},\left(I-n_{s} n_{s}\right) \cdot\langle v \rho\rangle$ will be almost $\left(I-\mathbf{n}_{\mathrm{s}} \mathbf{n}_{\mathrm{s}}\right) \cdot\langle\rho\rangle \mathbf{v}^{+}$. Therefore the choice of $\eta$ equal to $\xi^{+}$follows.

A second assumption concerns the entropy fluxes $w^{+}$and $w^{-}$. These are taken equal to $(\mathbf{q} / T)^{+}$respectively $(\mathbf{q} / T)^{-}$, a well-known result from bulk thermodynamics (Müller, 1985).

The remaining contact layer quantities $\langle\rho\rangle,\langle\rho e\rangle,\langle\rho h\rangle$ and $\langle\rho s\rangle$ in the balances of mass, internal energy and entropy provoke the definition of mass-weighted contact layer quantities

$$
\rho_{\mathrm{s}}=\langle\rho\rangle, \quad e_{\mathrm{s}}=\rho_{\mathrm{s}}^{-1}\langle\rho e\rangle, \quad h_{\mathrm{s}}=\rho_{\mathrm{s}}^{-1}\langle\rho h\rangle, \quad s_{\mathrm{s}}=\rho_{\mathrm{s}}^{-1}\langle\rho s\rangle,
$$

i.e. the mass density, internal energy, entropy and entropy production of the contact layer, respectively. The contact layer free energy $f_{\mathrm{s}}=e_{\mathrm{s}}-T_{\mathrm{s}} h_{\mathrm{s}}$ is defined with the (non-mass) weighted contact layer temperature $T_{\mathrm{s}}=\langle T\rangle$. Then the balances of mass, momentum, energy and entropy result in

$$
\begin{gathered}
\rho_{\mathrm{s}}^{\bullet}+\rho_{\mathrm{s}} \nabla_{\mathrm{s}} \cdot \mathbf{u}_{\mathrm{s}}=\mathbf{0} \\
\mathbf{\Sigma}^{+} \cdot \mathbf{n}^{+}+\Sigma^{-} \cdot \mathbf{n}^{-}=\mathbf{0} \\
\rho_{\mathrm{s}} e_{\mathrm{s}}^{\bullet}=-\mathbf{n}^{+} \cdot\left(\mathbf{q}^{+}-\mathbf{v}^{+} \cdot \Sigma^{+}\right)-\mathbf{n}^{-} \cdot\left(\mathbf{q}^{-}-\mathbf{v}^{-} \cdot \mathbf{\Sigma}^{-}\right) \\
-\rho_{\mathrm{s}}\left(f_{\mathrm{s}}^{\bullet}+T_{\mathrm{s}}^{\bullet} h_{\mathrm{s}}\right)+\mathbf{n}^{+} \cdot\left(\mathbf{q}^{+}\left(\frac{T_{\mathrm{s}}-T^{+}}{T^{+}}\right)+\mathbf{v}^{+} \cdot \mathbf{\Sigma}^{+}\right)+\mathbf{n}^{-} \cdot\left(\mathbf{q}^{-}\left(\frac{T_{\mathrm{s}}-T^{-}}{T^{-}}\right)+\mathbf{v}^{-} \cdot \Sigma^{-}\right) \geqslant 0
\end{gathered}
$$

\section{INDEPENDENT VARIABLES AND CONSTITUTIVE EQUATIONS}

In this section, we consider constitutive quantities, constitutive relations for these quantities and the independent variables occurring in these relationships. Constitutive principles induce restrictions with respect to the initially general constitutive relations (Hunter, 1976; Müller, 1985).

In continuum thermodynamics the position vector and temperature, as functions of material coordinates and time, are generally accepted as independent variables in constitutive equations. It is characteristic of the indeperdent variables that, if they are known as functions of location and time, all other quantities of interest can be calculated. For the contact layer it is assumed that the following variables, as being functions of $\eta$ and $t$, may be chosen to compose the set of independent variables

$$
\mathbf{x}_{\mathrm{s}}, \mathbf{x}^{+}, \mathbf{x}^{-}, T_{\mathrm{s}}, T^{+}, T^{-} \text {. }
$$

The contact layer mass density $\rho_{\mathrm{s}}$ is omitted as an independent variable as it can be expressed in $x_{\mathrm{s}}$ using the mass balance.

In the remaining thermodynamical balances, a set of quantities is present for which constitutive equations are needed. These dependent variables are

$$
f_{\mathrm{s}}, h_{\mathrm{s}}, q_{\mathrm{n}}^{+}, q_{\mathrm{n}}^{-}, \sigma^{+}, \sigma^{-}
$$

where the normal heat fluxes $q_{n}^{+}, q_{n}^{-}$are defined by $q_{n}^{+}=n^{+} \cdot q^{+}$, respectively $q_{n}^{-}=n^{-} \cdot q^{-}$, and the stress vectors $\sigma^{+}, \sigma^{-}$by $\sigma^{+}=n^{+} \cdot \Sigma^{+}$, respectively $\sigma^{-}=n^{-} \cdot \Sigma^{-}$. The normal heat fluxes are related to each other by the energy balance, the stress vectors by the action and reaction law. Therefore a constitutive equation is only needed for $\sigma^{+}$and $q_{n}^{+}$, whereas $\sigma^{-}$ and $q_{\mathrm{n}}^{-}$are dictated by the already mentioned balance laws.

The principles of determination, equipresence and local action are applied in order to arrive at constitutive equations. These result in the statement that each of the variables of 
(23) can be expressed in the history of the deformational quantities $\boldsymbol{F}_{\mathrm{s}}, \boldsymbol{F}^{+}, \boldsymbol{F}^{-} . \boldsymbol{\delta}_{\mathrm{i}}, \boldsymbol{\delta}^{+}$and $\delta^{-}$, and the thermal quantities $\mathbf{g}_{\mathrm{s}}, \mathbf{g}^{+}, \mathbf{g}^{-}, T_{\mathrm{s}}, T^{+}$and $T^{-}$. The deformation tensors $\boldsymbol{F}_{\mathrm{s}}$, $\boldsymbol{F}^{+}$and $\boldsymbol{F}^{-}$, defined by

$$
\boldsymbol{F}_{\mathrm{s}}=\left(\frac{\partial \mathbf{x}_{\mathrm{s}}}{\partial \underline{\eta}}\right)^{\mathrm{T}} \chi_{\mathrm{s} 0}, \quad \boldsymbol{F}^{+}=\left(\frac{\partial \mathbf{x}^{+}}{\partial \zeta^{+}}\right)^{\mathrm{T}} \chi_{0}^{+}, \quad \boldsymbol{F}^{-}=\left(\frac{\partial \mathbf{x}^{-}}{\partial \zeta^{-}}\right)^{\mathrm{T}} \chi_{0}^{-},
$$

take the non-material in-plane deformation of the contact layer and the material deformation of the bulk surfaces into account. Here $\chi_{50}, \chi_{0}^{+}$and $\chi_{0}^{-}$are the reciprocal vector bases in some reference state belonging, respectively to the surfaces $S, \mathrm{~S}^{+}$and $\mathrm{S}^{-}$. The deformation vectors $\delta_{\mathrm{s}}, \delta^{+}$and $\delta^{-}$, defined by

$$
\begin{gathered}
\delta_{\mathrm{s}}=\delta_{\mathrm{s} 0}+\int_{t_{0}}^{t}\left(\mathbf{v}^{+}(\eta, \tau)-\mathbf{v}^{-}(\eta, \tau)\right) \mathrm{d} \tau, \quad \delta^{+}=\delta_{0}^{+}+\int_{t_{10}}^{t}\left(\mathbf{v}^{+}\left(\zeta^{+}, \tau\right)-\mathbf{v}^{-}\left(\xi^{+}, \tau\right)\right) \mathrm{d} \tau, \\
\delta^{-}=\delta_{0}^{-}+\int_{r_{0}}^{t}\left(\mathbf{v}^{+}\left(\zeta^{-}, \tau\right)-\mathbf{v}^{-}\left(\zeta^{--}, \tau\right)\right) \mathrm{d} \tau
\end{gathered}
$$

account for thickness deformations and slip, which may be considered as extreme shear deformation of the contact layer. The time integrations start at time $t_{0}$ assigned to the reference state and end at the current time $t$. Finally the temperature gradients $\mathbf{g}_{s}, \mathbf{g}^{+}$and $\mathrm{g}^{-}$are defined by

$$
\mathbf{g}_{\mathrm{s}}=\nabla_{\mathrm{s}} T_{\mathrm{s}}, \quad \mathbf{g}^{+}=\nabla_{\mathrm{s}} T^{+}, \quad \mathbf{g}^{-}=\nabla_{\mathrm{s}} T^{-}
$$

History variables, also called hidden variables, are introduced to take the history of the independent variables into account. Based on the principle of equipresence, the time derivatives of these variables are given by evolution laws, in which only the current values of the independent variables and the hidden variables appear. By analogy with the foregoing, history variables $\underline{\sim}_{s}(\eta, t)$ of the contact layer and history variables $\underline{H}^{+}\left(\zeta^{+}, t\right), \underline{H}^{-}\left(\zeta^{-}, t\right)$ of the surfaces $\mathrm{S}^{+}$and $\mathrm{S}^{-}$, respectively are introduced.

Summarizing, each constitutive quantity $C(\eta, t)$ of $(23)$ is assumed to be a function of the actual values of the independent variables in (22) appropriate to contact layer point $\eta$, augmented by the actual values of the history variables pertaining to $\eta$ (with which a $\zeta^{+}$ and $\zeta^{-}$are always associated, see Section 2 ), so that

$$
C=C\left(\boldsymbol{F}_{\mathrm{s}}, \boldsymbol{F}^{+}, \boldsymbol{F}^{-}, \boldsymbol{\delta}_{\mathrm{s}}, \boldsymbol{\delta}^{+}, \boldsymbol{\delta}^{-}, \mathbf{g}_{\mathrm{s}}, \mathbf{g}^{+}, \mathbf{g}^{-}, T_{\mathrm{s}}, T^{+}, T^{-}, \underset{\sim}{H},{\underset{\sim}{H}}^{+},{\underset{\sim}{H}}^{-}\right) .
$$

The following types of equations have application as evolution laws for the history variables

$$
\begin{aligned}
& \underline{\sim}_{\mathrm{s}}^{\bullet}={\underset{\sim}{\mathrm{H}}}_{\mathrm{s}}^{\bullet}\left(\boldsymbol{F}_{\mathrm{s}}, \ldots, \underline{H}^{-}\right), \quad \underline{\sim}^{+}=\left.\frac{\partial \underline{H}^{+}}{\partial t}\right|_{\underline{\Sigma}^{+}}=\underline{\dot{H}}^{+}\left(\boldsymbol{F}_{\mathrm{s}}, \ldots, \underline{\sim}^{-}\right),
\end{aligned}
$$

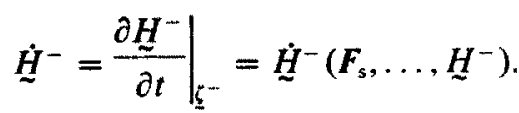

For an analysis of contact behaviour in the restricted class, it is unnecessary to consider all the independent variables in (27) and (28). It is assumed that only independent variables of the contact layer, together with $T^{+}$and $T^{-}$, are relevant for the constitutive equations to be constructed. It is also assumed that the coordinate system of the surface of the irreversible deforming body may be chosen for the layer's coordinate system $\eta$, without conflicting with Section 4 . Thus the eqns (27) and (28), by arbitrarily choosing $B^{+}$as the deforming body, simplify to 


$$
\begin{gathered}
C\left(\underline{\zeta}^{+}, t\right)=C\left(\boldsymbol{F}_{\mathrm{s}}, \boldsymbol{\delta}_{\mathrm{s}}, \mathbf{g}_{\mathrm{s}}, T_{\mathrm{s}}, T^{+}, T^{-}, \underset{\sim}{H}\right), \quad C \in\left\{f_{\mathrm{s}}, h_{\mathrm{s}}, q_{\mathrm{n}}^{+}, \sigma^{+}\right\}, \\
\dot{H}_{\mathrm{s}}\left(\zeta^{+}, t\right)=\left.\frac{\partial \underline{\sim}_{\mathrm{s}}}{\partial t}\right|_{\xi^{+}}=\dot{\sim}_{\mathrm{s}}\left(\boldsymbol{F}_{\mathrm{s}}, \boldsymbol{\delta}_{\mathrm{s}}, \mathbf{g}_{\mathrm{s}}, T_{\mathrm{s}}, T^{+}, T^{-},{\underset{\sim}{\mathrm{s}}}_{\mathrm{s}}\right) .
\end{gathered}
$$

Before the implications of the above for the entropy inequality are studied in the next section, a final constitutive principle will be applied, in this case the principle of objectivity. It states that constitutive equations should be invariant for observer transformations. Part of the principle is the assumption that mass density, stress vector, heat flux vector, internal energy, entropy, temperature and heat sources are objective quantities. The principle can be elaborated by introducing two different observers with the same notion of time and distance, e.g. see Müller (1985). Invariant or corotational quantities (Van Wijngaarden, 1988) can be used in order to obey objectivity of the constitutive equations. In Appendix $C$, a non-singular second-order tensor $A$, depending only on kinematic quantities, is introduced to transform objective quantities into invariant ones. The choice for $A$ is also considered there. It is found convenient to introduce the invariant stress vector $\hat{\sigma}^{+}$and deformation vector $\hat{\delta}_{\mathrm{s}}$ by means of

$$
\hat{\sigma}^{+}=\frac{\rho_{\mathrm{s} 0}}{\rho_{\mathrm{s}}} A^{-1} \cdot \sigma^{+}, \quad \hat{\delta}_{\mathrm{s}}=A^{\mathrm{c}} \cdot\left(\mathrm{v}^{+}-\mathrm{v}^{-}\right), \quad \hat{\delta}_{\mathrm{s}}\left(t_{0}\right)=0 .
$$

Instead of $F_{\mathrm{s}}$, the invariant contact layer right Cauchy-Green tensor $C_{\mathrm{s}}=\boldsymbol{F}_{\mathrm{s}}^{\mathrm{c}} \cdot \boldsymbol{F}_{\mathrm{s}}$ can be used in the constitutive equations as well as suitable invariant history variables $\hat{H}_{s}$ and $\hat{\mathbf{g}}_{\mathrm{s}}=\boldsymbol{A}^{\mathrm{i}} \cdot \mathbf{g}_{\mathrm{s}}$. This results in

$$
\begin{gathered}
\hat{C}=\hat{C}\left(C_{\mathrm{s}}, \hat{\boldsymbol{\delta}}_{\mathrm{s}}, \hat{\mathrm{g}}_{\mathrm{s}}, T_{\mathrm{s}}, T^{+}, T^{-}, \hat{H}_{\mathrm{s}}\right), \quad \hat{C} \in\left\{f_{\mathrm{s}}, h_{\mathrm{s}}, q_{\mathrm{n}}^{+}, \hat{\sigma}^{+}\right\}, \\
\hat{H}_{\mathrm{s}}=\hat{H}_{\mathrm{s}}\left(C_{\mathrm{s}}, \hat{\boldsymbol{\delta}}_{\mathrm{s}}, \hat{\mathrm{g}}_{\mathrm{s}}, T_{\mathrm{s}}, T^{+}, T^{-}, \hat{H}_{\mathrm{s}}\right) .
\end{gathered}
$$

\section{THE ENTROPY INEQUALITY}

The implications of the entropy inequality, based on the results of the previous section, are discussed here. With the invariant quantities, it follows from eqn (21) that

$$
-\rho_{\mathrm{s} 0}\left(f_{\mathrm{s}}+\dot{T}_{\mathrm{s}} h_{\mathrm{s}}\right)+\frac{\rho_{\mathrm{s} 0}}{\rho_{\mathrm{s}}}\left(q_{\mathrm{n}}^{+} \frac{T_{\mathrm{s}}-T^{+}}{T^{+}}+q_{\mathrm{n}}^{-} \frac{T_{\mathrm{s}}-T^{-}}{T^{-}}\right)+\hat{\sigma}^{+} \cdot \hat{\delta}_{\mathrm{s}} \geqslant 0
$$

As usual, it is assumed that decomposition into the inequalities

$$
\begin{aligned}
& -\rho_{\mathrm{s} 0}\left(\dot{f}_{\mathrm{s}}+\dot{T}_{\mathrm{s}} h_{\mathrm{s}}\right)+\hat{\sigma}^{+} \cdot \hat{\delta}_{\mathrm{s}} \geqslant 0, \\
& q_{\mathrm{n}}^{+} \frac{T_{\mathrm{s}}-T^{+}}{T^{+}}+q_{\mathrm{n}}^{-} \frac{T_{\mathrm{s}}-T^{-}}{T^{-}} \geqslant 0
\end{aligned}
$$

is allowed. The second inequality restricts the possible heat fluxes perpendicular to the contact layer. Simple constitutive expressions for $q_{\mathrm{n}}^{+}$and $q_{\mathrm{n}}^{-}$are given by

$$
\begin{array}{ll}
q_{\mathrm{a}}^{+}=\alpha^{+}\left(T_{\mathrm{s}}-T^{+}\right), & \alpha^{+}>0, \\
q_{\mathrm{n}}^{-}=\alpha^{-}\left(T_{\mathrm{s}}-T^{-}\right), & \alpha^{-}>0 .
\end{array}
$$




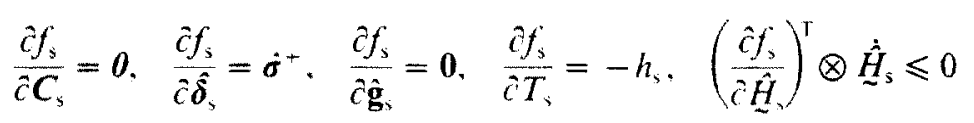

where 0 is the second-order zero tensor and $\otimes$ an operator determined by the character of the different constituents of $\hat{\sim}_{\text {s. }}$. The result (39) states that $f_{s}$ is independent of the in-plane deformations of the contact layer and, consequently, that the contact stresses are not influenced by these deformations. This is felt to be a shortcoming in the presented theory. Deformations of the surface of the formed body cause changes in roughness. which affect the stiffness of the contact layer and the frictional behaviour. In-plane contact-layer stresses (a flux neglected in Section 3) unequal zero are needed to deal with such influences, a case which is not elaborated here. The result (39) for the contact stresses $\hat{\sigma}^{+}$is valid only if $\hat{H}_{\text {s }}$ is independent of $\hat{\delta}_{\mathrm{s}}$. This will be the case in what follows. The history parameters are further restricted by the remaining inequality of (39).

\section{MAXWELL FRICTION MODELS}

In this section a special choice of history variables yields a constitutive equation for the contact stress vector, which resembles the constitutive equation for elastoplastic material behaviour. This method was described for bulk-material behaviour by Van Wijngaarden (1988). The resulting equations are analogous to those given, for instance by Cheng and Kikuchi (1985) and Baaijens (1987).

The contact layer's free energy is given by

$$
f_{\mathrm{s}}=f_{\mathrm{s}}\left(\hat{\delta}_{\mathrm{s}}-\hat{\delta}_{\mathrm{s}}^{\mathrm{ir}}, T_{\mathrm{s}}, H_{\mathrm{t}}, H_{\mathrm{n}}\right)
$$

in which $H_{\mathrm{t}}$ and $H_{\mathrm{n}}$ are scalar history variables. The vector quantity $\hat{\delta}_{\mathrm{s}}^{\mathrm{ir}}$ is the irreversible contact layer deformation vector, a first-order history variable. In (40) it is assumed that only the reversible part $\hat{\boldsymbol{\delta}}_{\mathrm{s}}^{\mathrm{r}}=\hat{\boldsymbol{\delta}}_{\mathrm{s}}-\hat{\boldsymbol{\delta}}_{\mathrm{s}}^{\mathrm{ir}}$ of $\hat{\boldsymbol{\delta}}_{\mathrm{s}}$ is of interest for the free energy. This assumption is similar to decomposition into the elastic (reversible) and plastic (irreversible) deformation in bulk mechanics (Lee, 1969: Sidoroff, 1973). Note that, in normal and in-plane (or slip) direction, reversible and irreversible deformation of the contact layer is possible. Evolution laws for $\hat{\delta}_{\mathrm{s}}^{\text {ir }}, \dot{H}_{\mathrm{t}}$ and $\dot{H}_{\mathrm{n}}$ as functions of $\hat{\delta}_{\mathrm{s}}-\hat{\delta}_{\mathrm{s}}^{\mathrm{ir}}, T_{\mathrm{s}}, H_{\mathrm{t}}$ and $H_{\mathrm{n}}$ are needed. For $\hat{\delta}_{\mathrm{s}}^{\mathrm{ir}}$, it is assumed that a potential $\varphi=\varphi\left(\hat{\delta}_{\mathrm{s}}-\hat{\boldsymbol{\delta}}_{\mathrm{s}}^{\mathrm{ir}}, T_{\mathrm{s}}, H_{\mathrm{t}}, H_{\mathrm{n}}\right)$ or equivalently $\varphi=\varphi\left(\dot{\sigma}^{-} . T_{\mathrm{s}}, H_{\mathrm{t}}, H_{\mathrm{n}}\right)$ exists, so that

$$
\hat{\delta}_{\mathrm{t}}^{\mathrm{ir}}=\frac{\partial \varphi}{\partial \hat{\tau}}, \quad \dot{\hat{\delta}_{\mathrm{n}}^{\mathrm{ir}}}=\frac{\partial \varphi}{\partial \hat{\mathbf{p}}}
$$

where the invariant tangential or frictional stress vector $\hat{\tau}$, the normal or compressive stress vector $\hat{\mathbf{p}}$, the irreversible tangential displacement or slip vector $\delta_{\imath}^{\mathrm{ir}}$ and the irreversible normal displacement or compression vector $\hat{\delta}_{\mathrm{n}}^{\mathrm{ir}}$ are defined by

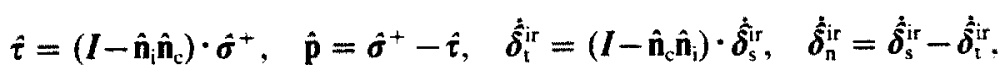

$$
\begin{aligned}
& \hat{\mathbf{n}}_{\mathbf{i}}=A^{-1} \cdot \mathbf{n}^{+}, \quad \hat{\mathbf{n}}_{\mathrm{c}}=A^{\mathfrak{c}} \cdot \mathbf{n}^{+} \text {. }
\end{aligned}
$$

In an isotropic case (41) can be simplified to

$$
\dot{\hat{\delta}_{\mathrm{t}}}=\frac{\hat{\tau}}{\tau} \frac{\partial \varphi}{\hat{c} \tau}, \quad \hat{\boldsymbol{\delta}}_{\mathrm{n}}^{\mathrm{r}}=\frac{\hat{\mathbf{p}}}{p} \frac{\partial \varphi}{\partial p}, \quad \varphi=\varphi\left(\tau, p, T_{\mathrm{s}}, H_{\mathrm{t}}, H_{\mathrm{n}}\right)
$$

with $\tau=\|\hat{\imath}\|$ and $p=\|\hat{\mathbf{p}}\|$. It is assumed that $f_{\mathrm{s}}$ is given by 


$$
f_{\mathrm{s}}=\frac{1}{2}\left(\hat{\delta}_{\mathrm{t}}^{\mathrm{r}} \cdot S_{\mathrm{t}} \cdot \hat{\delta}_{\mathrm{t}}^{\mathrm{r}}+\hat{\delta}_{\mathrm{n}}^{\mathrm{r}} \cdot \hat{\delta}_{\mathrm{n}}^{\mathrm{r}} S_{\mathrm{n}}\right)+\psi\left(T_{\mathrm{s}}, H_{\mathrm{t}}, H_{\mathrm{n}}\right)
$$

where $S_{\mathrm{t}}$ and $s_{\mathrm{n}}$ denote a constant stiffness tensor and scalar, respectively. A combination of (39) and the above equations provides a constitutive Maxwell relationship for the invariant tangential and normal stress vector

$$
\begin{aligned}
& \frac{1}{\rho_{\mathrm{s} 0}} \dot{\boldsymbol{\tau}}+S_{\mathrm{t}} \cdot \frac{\hat{\boldsymbol{\tau}}}{\tau} \frac{\partial \varphi}{\partial \tau}=S_{\mathrm{t}} \cdot \dot{\boldsymbol{\delta}}_{\mathrm{t}}, \\
& \frac{1}{\rho_{\mathrm{s} 0}} \dot{\hat{\mathbf{p}}}+S_{\mathrm{n}} \frac{\hat{\mathbf{p}}}{p} \frac{\partial \varphi}{\partial p}=S_{\mathrm{n}} \hat{\boldsymbol{\delta}}_{\mathrm{n}} .
\end{aligned}
$$

If $A$ is a rotation tensor and $S_{\mathrm{t}}=s_{\mathrm{t}} I$, the above relationships can be formulated as

$$
\begin{aligned}
& \frac{1}{\rho_{\mathrm{s} 0}}\left(\dot{\tau}+\boldsymbol{A} \cdot\left(\dot{A}^{-1}\right) \cdot \tau-\frac{\dot{\rho}_{\mathrm{s}}}{\rho_{\mathrm{s}}} \tau\right)+s_{\mathrm{t}} \frac{\tau}{\tau} \frac{\partial \varphi}{\partial \tau}=\left(\frac{\rho_{\mathrm{s} 0}}{\rho_{\mathrm{s}}}\right)^{-1} s_{\mathrm{t}} \dot{\boldsymbol{\delta}}_{\mathrm{t}}, \\
& \frac{1}{\rho_{\mathrm{s} 0}}\left(\dot{\mathrm{p}}+\boldsymbol{A} \cdot\left(\dot{A}^{-1}\right) \cdot \mathbf{p}-\frac{\dot{\rho}_{\mathrm{s}}}{\rho_{\mathrm{s}}} \mathrm{p}\right)+s_{\mathrm{n}} \frac{\mathbf{p}}{p} \frac{\partial \varphi}{\partial p}=\left(\frac{\rho_{\mathrm{s} 0}}{\rho_{\mathrm{s}}}\right)^{-1} s_{\mathrm{n}} \dot{\boldsymbol{\delta}}_{\mathrm{n}}
\end{aligned}
$$

with $\tau=\left(I-\mathbf{n}^{+} \mathbf{n}^{+}\right) \cdot \sigma^{+}, \mathbf{p}=\sigma^{+}-\tau, \boldsymbol{\delta}_{\mathrm{t}}=\left(I-\mathbf{n}^{+} \mathbf{n}^{+}\right) \cdot \boldsymbol{\delta}_{\mathrm{s}}$ and $\boldsymbol{\delta}_{\mathrm{a}}=\boldsymbol{\delta}_{\mathrm{s}}-\boldsymbol{\delta}_{\mathrm{t}}$. Using the mass balance, $\dot{\rho}_{\mathrm{s}} / \rho_{\mathrm{s}}$ can be replaced by the surface magnification rate $\nabla_{\mathrm{s}} \cdot \mathrm{v}^{+}$while $\rho_{\mathrm{s} 0} / \rho_{\mathrm{s}}$ can be identified as the surface magnification factor of the current state compared to the reference state. The combinations $i+A \cdot\left(\dot{A}^{-1}\right) \cdot \tau$ and $\dot{p}+\boldsymbol{A} \cdot\left(\dot{A}^{-1}\right) \cdot \mathrm{p}$ are objective rates $\tau$ and $\mathrm{p}$, respectively. Identification with such rates in literature (Baaijens et al., 1986) is possible (Starmans, 1989).

As for constitutive equations for metals, the history variables $H_{\mathrm{t}}$ and $H_{\mathrm{n}}$ introduce hardening (or softening). Irreversible work hardening is considered as an example. In that case $H_{\mathrm{t}}$ is identified as the irreversible frictional work and $H_{\mathrm{n}}$ as the irreversible compressive work

$$
H_{\mathrm{t}}=\int_{t_{0}}^{t} \hat{\tau} \cdot \hat{\delta_{\mathrm{t}}^{\mathrm{in}}} \mathrm{d} \tau, \quad H_{\mathrm{n}}=\int_{t_{0}}^{t} \hat{\mathbf{p}} \cdot \hat{\delta}_{\mathrm{n}}^{\hat{\mathrm{t}}} \mathrm{d} \tau .
$$

For their evolution laws, it follows with (41), that

$$
\dot{H}_{\mathrm{t}}=\tau \frac{\partial \varphi}{\partial \tau}, \quad \dot{H}_{\mathrm{n}}=p \frac{\partial \varphi}{\partial p}
$$

The inequality in (39) results in

$$
\left(\frac{\partial f_{\mathrm{s}}}{\partial H_{\mathrm{t}}}-1\right) \tau \frac{\partial \varphi}{\partial \tau}+\left(\frac{\partial f_{\mathrm{s}}}{\partial H_{\mathrm{n}}}-1\right) p \frac{\partial \varphi}{\partial p} \leqslant 0
$$

If the deformation of the contact layer in the thickness direction is unimportant or negligible, the normal part of the deformation vector disappears as an independent variable in the constitutive equations. The normal stress vector $\hat{\mathbf{p}}$, whose constitutive equation (46) is then cancelled, becomes an independent variable instead. Consideration of the entropy inequality results in the conclusion that $f_{\mathrm{s}}$ has to be independent of $\hat{\mathbf{p}}$. The only influence of $\hat{\mathbf{p}}$ on $\hat{\boldsymbol{t}}$ then remaining is that via $\varphi$, which is not restricted in any way. Hence reversible shear behaviour cannot be influenced by the normal stress and the observations made on (39) in Section 6 apply here as well. 


\section{CONCLUDING REMARKS}

In the foregoing, contact behaviour in a continuum formulation is discussed. Mechanical constitutive contact equations consistent with formulated thermodynamic theory have been derived. Coulomb and Von Mises like friction laws are not rejected, as they are special cases of these constitutive equations (Baaijens, 1987). If greater restrictions for the constitutive equations are to be obtained, the different thermodynamic quantities have to be specified in more detail. For such a specification, which cannot be gone into here, results obtained from structural modelling of real contacts (e.g. Francis, 1977; Landheer et al., 1980; Tangena, 1987) can be used. As contact models on a microscopic scale are needed for such an approach, research results on the microscopic physical behaviour of contacting surfaces are also indispensable if more restrictive statements are to be obtained.

The constitutive equations given are derived from numerous simplifications and assumptions. Whether such equations can describe real contact behaviour will have to be evaluated by experiments in which detailed data are obtained for all contact quantities of importance. For the upsetting test, Starmans (1989) described some methods for determining the relative displacements in the contact area between tool and test piece, as well as the contact stresses. It is to be expected that the application of the described constitutive equations in the upsetting configurations can be evaluated by these methods.

\section{REFERENCES}

Baaijens, F. P. T. (1987). On a numerical method to solve contact problems. Ph.D. Thesis, Eindhoven University of Technology, The Netherlands.

Baaijens, F. P. T., Brekelmans, W. A. M., Veldpaus, F. E. and Starmans, F. J. M. (1986). A constitutive equation for frictional phenomena including history dependency. In Proc. 2nd International Conf. on Numerical Methods in Industrial Forming Processes (Edited by K. Mattiasson. A. Samuelsson, R. D. Wood and O. C. Zienkiewicz), pp. 91-96. Balkema, Rotterdam.

Bikerman, J. J. (1970). Thermodynamics, adhesion and sliding friction. J. Lubric. Technol. Trans. ASME 92 , 243-247.

Cheng, J.-H. and Kikuchi, N. (1985). An incremental constitutive relation of unilateral contact friction for large deformation analysis. J. Appl. Mech. Trans. ASME 52,639-648.

Francis, H. A. (1977). Application of spherical indentation mechanics to reversible and irreversible contact between rough surfaces. Wear $45,221-269$.

Fredriksson, B. (1976). Finite element solution of surface nonlinearities in structural mechanics with special emphasis to contact and fracture mechanics problems. Comput. Struct. 6, 281-290.

Ilunter, S. C. (1976). Mechanics of Continuous Media. John Wiley, New York.

Huyghe, J. M. R. J. (1986). Non-linear finite element models of the beating left ventricle and the intramyocardial coronary circulation. Ph.D. Thesis, Eindhoven University of Technology, The Netherlands.

Klamecki, B. E. (1980). A thermodynamical model of friction. Wear 63, 113-120.

Landheer, D., Dackus, A. J. G. and Klostermann, J. A. (1980). Fundamental aspects and technological implications of the solubility concept for the prediction of running properties. Wear 62, 255-286.

Lee, E. H. (1969). Elastic-plastic deformations at finite strain. J. Appl. Mech. Trans. ASME 36, 1-6.

Michalowski, R. and Mróz, Z. (1978). Associated and non-associated sliding rules in contact friction problems. Arch. Mech. 30, 259-276.

Müller, I. (1985). Thermodynamics. Pitman Publishing, London.

Oden, J. T. and Martins, J. A. C. (1985). Models and computational methods for dynamic friction phenomena. Comput. Methods Appl. Mech. Engng 52, 527-634.

Petersson, H. (1977). Constitutive Relations for Contacting Solids. Division of Structural Design, publication $77-$ 10. Chalmers University of Technology, Göteborg, Sweden.

Rabinowicz, E. (1965). Friction and Wear of Materials. John Wiley, New York.

Ruina, A. L. (1985). Constitutive relations for frictional slip. Chapter 9 in Mechanics of Geomaterials (Edited by Z. Bažant). John Wiley, New York.

Sidoroff, F. (1973). The geometrical concept of intermediate configuration and elastic-plastic finite strain. Arch. Mech. 25, 299-309.

Starmans, F. J. M. (1989). On friction in forming. Ph.D. Thesis, Eindhoven University of Technology, The Netherlands.

Tangena, A. G. (1987). Tribology of thin film systems. Ph.D. Thesis, Eindhoven University of Technology, The Netherlands.

Van Wijngaarden, H. (1988). Constitutive equations for metals with an application to the extrusion of lead. Ph.D. Thesis, Eindhoven University of Technology, The Netherlands.

Whitaker, S. (1969). Advances in theory of fluid motion in porous media. Ind. Engng Chem. 61(12), 14-28.

Zmitrowicz. A. (1987). A thermodynamical model of contact friction and wear: I Governing equations; II Constitutive equations for materials and linearized theories; III Constitutive equations for friction, wear and frictional heat. Wear 114, 135-168; 114, 169-197; 114, 199-221. 


\section{APPENDIX A: THE GENERAL BALANCE LAW FOR CONTACT SURFACES}

To derive the balance law (10) for some mass-associated quantity $\varphi$, the averaging volume $V_{\mathrm{e}}$. belonging to a point $\eta$, is followed. The quantity $Q$, defined for this volume according to (6), is considered.

The change $Q^{\bullet}=A_{c}\langle\rho \varphi\rangle^{\bullet}$ of $Q$ in time originates from three causes, as follows.

(1) Material flow through the border $\partial V_{\mathrm{e}}$ of $V_{\mathrm{e}}$. The decrease in $Q$ per unit of time due to convection is

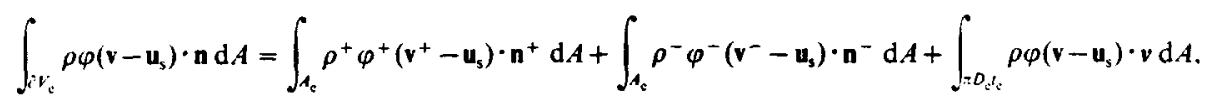

with $n^{+}$and $n^{-}$the unit outward normal vectors on $V_{c}$ at the $S^{+}$and $S^{-}$surfaces respectively, parallel to $n_{r}$, and $v$ the unit outward normal vector on the cylindrical surface of $V_{e}$, perpendicular to $n_{\text {s. }}$. The convection term is broken down to surface integrals for $\mathrm{S}^{+}$and $\mathrm{S}^{-}$and the cylindrical surface. The superseripts + and indicate bulk continuum quantities at the + and - sides of the averaging volume $V_{e}$. The continuum quantities at $S^{-}$ and $\mathrm{S}^{-}$vary with characteristic length $L$ along these surfaces. Therefore the integrals over $A_{\mathfrak{c}}$ may be written as

$$
\mathbf{n} \cdot\left(\mathbf{v}^{+}-\mathbf{u}_{\mathrm{s}}\right) \rho^{+} \varphi^{+} A_{\mathrm{e}}, \mathbf{n}^{-} \cdot\left(\mathrm{v}^{-}-\mathrm{u}_{\mathrm{s}}\right) \rho^{-} \varphi^{-} A_{\mathrm{c}} .
$$

The integral over the cylindrical surface of $V_{\epsilon}$ can be replaced, using Appendix B, by

$$
\left(\nabla_{s} \cdot\left[\left(I-n_{s} n_{s}\right) \cdot\langle v \rho \varphi\rangle\right]\right) A_{e}-u_{s} \cdot\left(\nabla_{s} \cdot\left[\left(I-n_{s} n_{s}\right)\langle\rho \varphi\rangle\right]\right) A_{e},
$$

with $\mathbf{n}_{\mathrm{s}}$ chosen equal to $\mathbf{n}^{+}$or $\mathbf{n}^{-}$arbitrarily. Expression (A3) can be reformulated as

$$
\left(\nabla_{s} \cdot\left[\left(I-n_{s} \mathbf{n}_{s}\right) \cdot\langle v \rho \varphi\rangle\right]\right) A_{\mathrm{s}}-\left(\nabla_{s} \cdot\left[\left(I-\mathbf{n}_{s} \mathbf{n}_{s}\right) \cdot \mathbf{u}_{s}\langle\rho \varphi\rangle\right]\right) A_{\mathrm{e}}+\left(\nabla_{s} \cdot \mathbf{u}_{s}\right) A_{c}\langle\rho \varphi\rangle
$$

(2) Flux through the boundary $\partial V_{\mathrm{e}}$. With $\psi$ indicating the local flux, the decrease in $Q$ resulting from fluxes is

$$
\int_{2 v_{\mathrm{s}}} \mathrm{n} \cdot \psi \mathrm{d} A=\int_{A_{\mathrm{c}}} \mathrm{n}^{+} \cdot \psi^{+} \mathrm{d} A+\int_{A_{\mathrm{c}}} \mathrm{n}^{-} \cdot \psi^{-} \mathrm{d} A+\int_{n D_{c^{2}}} \nu \cdot \psi \mathrm{d} A
$$

Likewise, the right-hand integrals may be transferred to, respectively,

$$
\mathrm{n}^{+} \cdot \psi^{+} A_{\mathrm{e}}, \quad \mathrm{n}^{-} \cdot \psi^{-} A_{\mathrm{e}}, \quad\left(\nabla_{\mathrm{s}} \cdot\left[\left(I-\mathrm{n}_{\mathrm{s}} \mathrm{n}_{\mathrm{s}}\right) \cdot\langle\boldsymbol{\psi}\rangle\right]\right) A_{\mathrm{e}} .
$$

(3) Sources within $V_{e}$. With the distributed mass-associated $\varphi$-production $\Phi$ as the only source, it holds, for the increase in $Q$, that

$$
\int_{r_{\mathrm{s}}} \rho \Phi \mathrm{d} V=A_{\mathrm{e}}\langle\rho \Phi\rangle
$$

Combining the above relations results in the general balance law (10), an equation only valid if the conditions ( 2$)$ for the dimensions of the averaging volume are satisfied.

\section{APPENDIX B : A SPECIAL SURFACE INTEGRAL} form

In this Appendix, surface integrals over the cylindrical surface of an elementary averaging volume $V_{\mathrm{e}}$ of the

$$
\int_{x D_{e^{\prime} e_{e}}} v \cdot q d A
$$

are elaborated, with $q$ a tensor of first or higher order and $v$ the unit outward normal vector. By separating the integration over the surface into an integration over the thickness $t_{\mathrm{e}}$ of $V_{\mathrm{e}}$ and the circumference $\pi D_{\mathrm{e}},(\mathrm{B} 1)$ can be reformulated as

$$
\int_{\pi D_{c}} v \cdot\left(\int_{l_{c}} q \mathrm{~d} h\right) \mathrm{d} l
$$

with $h$ a coordinate in the thickness direction and $l$ a coordinate in the circumferential direction. Assuming the integral of $q$ over $t_{\mathrm{e}}$ as smooth, so that the divergence theorem on the surface can be applied, it follows that

$$
\int_{x D_{e} e_{e}} v \cdot q d A=\int_{A_{e}} \nabla_{s} \cdot\left[\left(I-n, n_{s}\right) \cdot \int_{t_{e}} q \mathrm{~d} h\right] \mathrm{d} A
$$

From (2) and the definition of $\langle q\rangle$ according to (1) we have 


$$
\int_{\pi D_{s^{\prime} c_{c}}} v \cdot q \mathrm{~d} A=\left(\nabla_{s} \cdot\left[\left(I-\mathbf{n}_{s} \mathbf{n}_{s}\right) \cdot q\right]\right) A_{s} .
$$

A certain smoothness for the integral of $q$ in the thickness direction was assumed in order to derive (B4). The result suggests that this is too high a demand, as the right-hand side of (B4) is already properly defined if $\langle\boldsymbol{q}\rangle$ is continuously differentiable. According to the considerations of Section 2 . this demand for $\langle q\rangle$ is guaranteed for all relevant cases.

It likewise shows for a scalar quantity $q$ that

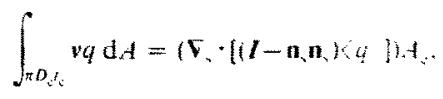

\section{APPENDIX C: SOME ASPECIS OF OBJECTIVITY}

The principle of frame indifference is first briefly discussed. Two observers $O$ and $O$, each with its own orthonormal vector base, are considered. The observers are assumed equivalent, i.e. with equal notion of time and distance. The time difference between $O$ and $O$ is arbitrarily chosen to equal zero. Quantities observed by observer $O$ are marked with a superscript bar, quantities observed by $O$ are not. For example. the position vector of some point $P$ in space. as observed by $O$. is indicated with $\mathbf{x}$. and as observed by $\bar{O}$ with $\bar{x}$. The components of some vectorial quantity $\mathbf{v}$ with respect to the orthonormal vector base $\left\{\mathbf{e}_{1}, \mathbf{e}_{2}, \mathbf{e}_{3} ;\right.$ of $\mathrm{O}$ combine to column $\underline{v}$, called the columnar representation of $v$ with respect to $\left.e_{1}, e_{2}, e_{3}\right\}$. Equivalently, the components of $i$ with respect to the orthonormal vector base $\left\{\overline{\mathbf{e}}_{1}, \overline{\mathbf{e}}_{2}, \overline{\mathbf{e}}_{3}\right\}$ of $\bar{O}$ combine to the columnar representation $\vec{t}$. In the same way, some second-order tensorial quantity $T$ has a matrix representation $T$ with respect to $e_{i}, e_{2}, e_{i}$ and $T$ a matrix representation $\underline{T}$ with respect to $\left\{\overrightarrow{\mathbf{e}}_{1}, \overline{\mathbf{e}}_{2}, \dot{\mathbf{e}}_{3}\right\}$.

The coordinates $x$ and $\bar{x}$ of some point $P$ in space are related by Euelidian transformations. The transformation expressing $\vec{x}$ in $x$ reads

$$
\underline{x}(t)=\underline{Q}(t) \underline{x}(t)+\underline{u}(t)
$$

with $\bar{Q}$ a time-dependent orthonormal tensor and $\tilde{\mathbf{u}}$ a time-dependent vector. For an arbitrary time $t, \bar{Q}$ and $\tilde{\mathbf{u}}$ indicate the rotation and the transiation, respectively of $O$ with respect to $O$. Physical quantities are called objective if they are related in a defined way. For scalar, vector and second-order tensor quantities the defining relations are

$$
\bar{B}=B, \quad \underline{\bar{B}}=\underline{Q} \underline{B} . \quad \underline{\bar{B}}=\overline{\bar{Q}} \underline{B}_{\underline{\underline{W}}}^{\mathrm{T}} .
$$

A physical quantity is called invariant if it is observed as being identical by two equivalent observers. A scalar objective quantity is also invariant. The principle of frame indifference states that constitutive equations should not change due to observer transformations. This is the case if they cuntain only objective or only invariant terms. Part of the principle is also the assumption that mass density, stress and force vector, heat fux vector. internal energy, entropy and heat sources are objective. It is noted that the balance laws of Section 4 do not change due to observer transformations, except for the part $n^{+} \cdot \Sigma^{+} \cdot v^{+}-n^{-} \cdot \Sigma^{-} \cdot v^{-}$, which can be formulated as $\sigma^{+} \cdot\left(v^{+}-v^{-}\right)$ using (19) and $\sigma^{+}=n^{+} \cdot \Sigma^{+}$. The stress vector $\sigma^{+}$is objective, the velocity difference is only objective in a restricted sense. For this velocity difference the observations of $O$ and $O$ are related by

$$
\underline{t}^{+}-\underline{t}^{-}=\underline{Q}(t)\left(x^{+}-\underline{x}^{-}\right)+\underline{Q}(t)\left(\underline{t}^{-}-\underline{t}^{-}\right)
$$

Since $t_{\mathrm{e}} \ll L$, the first term on the right-hand side is negligible, resulting in an equation satisfying objectivity, but only for transformations for which $Q(t)=0$ objectivity is specifically guaranteed.

An invariant stress vector and contact layer deformation vector are introduced in Section 5 using some second-order tensor $A=A(\eta, t)$. With the foregoing, it shows that second-order tensors $A$ can be used which obey the relationship

$$
\underline{A}(\eta, t)=\bar{Q}(t) A(\eta, t), \quad \operatorname{det}(\underline{-1}) \neq 0
$$

with det $(A)$ the determinant of $A$. So as not to conflict with the accepted independent variables of Section 5 , only tensors $A$ containing kinematics of the surfaces $S, S^{+}$and $S^{-}$are considered. An example of $A$. obeying (C4), is a linear combination of the in-plane deformation tensors $\boldsymbol{F}_{\mathrm{s}}, \boldsymbol{F}^{+}, \boldsymbol{F}^{-}$plus a contribution $\mathbf{n}_{\mathrm{s}} \mathbf{n}_{\mathrm{s},}$ accounting for the rotation of the direction normal to the contact layer, with the subscript 0 referring to the reference state chosen. so that

$$
A=x_{s} F_{s}+z^{+} F^{+}+x^{-} F^{-}+\beta \mathbf{n}, \mathbf{n}_{\mathrm{s} i} \text {. }
$$

The coefficients $\alpha_{5}, \alpha^{+}, \alpha^{-}$and $\beta$ may be functions of the surface change factors $\nabla_{50} \cdot x_{5,}, \nabla_{50} \cdot x^{-}$and $\nabla_{50} \cdot x^{-}$. In the case that only quantities belonging to the surface $S^{+}$are considered of importance, the following choices are legitimate

$$
\boldsymbol{x}^{+} \boldsymbol{F}^{+}+\beta \mathbf{n}^{+} \mathbf{n}_{0}^{+}, \quad \boldsymbol{x}^{+}\left(\boldsymbol{F}^{+}\right)^{-\mathrm{c}}+\beta \mathbf{n}^{+} \mathbf{n}_{0}^{+}, \quad \boldsymbol{x}^{-} \boldsymbol{R}^{+}+\beta \mathbf{n}^{+} \mathbf{n}_{0}^{+}
$$

with $\boldsymbol{R}^{+}$such that $\boldsymbol{F}^{+}=\boldsymbol{R}^{+} \cdot \boldsymbol{U}^{+}$with

$$
\left(U^{+}\right)^{+}=U^{+}, \quad R^{+} \cdot\left(R^{+}\right)^{\mathrm{e}}=\boldsymbol{l}-\mathbf{n}^{+} \mathbf{n}^{+}, \quad \operatorname{det}\left(\boldsymbol{R}^{+}+\mathbf{n}^{+} \mathbf{n}_{0}^{+}\right)=1 .
$$

